九州大学学術情報リポジトリ

Kyushu University Institutional Repository

\title{
A Study on Smoothed Particle Hydrodynamics for Liquid Metal Flow Simulation
}

Al Salami, Jabir

Interdisciplinary Graduate School of Engineering Sciences, Kyushu University

Hu, Changhong

Faculty of Research Institute of Applied Mechanics, Kyushu University : Professor

Hanada, Kazuaki

Faculty of Research Institute of Applied Mechanics, Kyushu University : Professor

https://doi.org/10.5109/2349294

出版情報：Evergreen. 6 (3)，pp.190-199，2019-09. 九州大学グリーンテクノロジー研究教育センター バージョン：

権利関係 : 


\title{
A Study on Smoothed Particle Hydrodynamics for Liquid Metal Flow Simulation
}

\author{
Jabir Al Salami ${ }^{*}$, Changhong $\mathrm{Hu}^{2}$, Kazuaki Hanada ${ }^{2}$ \\ ${ }^{1}$ Interdisciplinary Graduate School of Engineering Sciences, Kyushu University, Japan \\ ${ }^{2}$ Faculty of Research Institute of Applied Mechanics, Kyushu University, Japan \\ *Author to whom correspondence should be addressed, \\ E-mail: jsalami@riam.kyushu-u.ac.jp
}

(Received December 6, 2018; accepted July 12, 2019).

\begin{abstract}
In order to address the problem of heat and particle removal in tokamak-type, magnetic confinement nuclear fusion reactors, a divertor that utilizes liquid metal is suggested to replace solid tungsten divertors due to concerns regarding their structural integrity at high energy fluxes. The operation of such a device gives rise to phenomena spanning multiple disciplines of physics such as fluid dynamics, electromagnetics, thermodynamics and plasma physics. Smoothed Particle Hydrodynamics (SPH) is a Lagrangian, mesh-free numerical method that has been proven effective in a variety of disciplines. In this work, the hydrodynamic aspect of the liquid metal divertor is simulated using SPH, paving the way to implement additional physics in future work.
\end{abstract}

Keywords: Smoothed Particle Hydrodynamics, Liquid Metal.

\section{Introduction}

Nuclear fusion, the reaction that powers the stars in our universe, is a potential source of zero-carbon-emissions, highly safe and abundant energy here on the Earth. In a fusion reactor, Deuterium and Tritium are fused at temperatures in excess of tens of millions of degrees to produce energy. Because no known material can withstand and contain temperatures anywhere near those needed to achieve nuclear fusion, the core plasma in a tokamak-type fusion reactor is contained by means of a strong magnetic field to avoid direct contact with the solid walls of the reaction chamber. An essential component of the tokamak reactor is the divertor, which is responsible for expelling reaction byproducts and unspent fuel that adversely affect the plasma performance in addition to help extract energy from the reaction to produce usable energy.

One of the main challenges facing divertor design is the potential damage to solid plasma facing components, primarily made from tungsten due to the extremely high thermal fluxes $\left(\sim 10 \mathrm{MW} / \mathrm{m}^{2}\right)$ anticipated in future designs of reactors planned to provide energy to the public electricity grid $^{1,2)}$. Liquid metals were suggested to replace solid plasma facing components as viable alternatives for future fusion reactor designs ${ }^{20,21,22)}$.

Smoothed Particle Hydrodynamics is a Computational Fluid Dynamics (CFD) method that meets the requirements to be able to model multi-physics phenomena similar to the case with the liquid metal divertor ${ }^{23,24,25)}$. In particular, Smoothed Particle
Hydrodynamics (SPH) is better adapted and is easier to implement in cases of highly non-linear phenomena, such as free-surface flows, splashing and fragmentation when compared to its Eulerian counterparts due to its purely mesh-free, Lagrangian nature ${ }^{14,25)}$. Furthermore, the SPH framework facilitates the integration of multi-physics without the need for extensive and computationally demanding and complicated approaches aiming to resolve free-surfaces and non-hydrodynamical phenomena. In this work, the hydrodynamical aspect of the operation of a liquid metal divertor tank is simulated using SPH, where liquid metal is circulated in a tank as it is being bombarded by plasma particles from above. A key desired simulation outcome is the extent and speed at which new fluid is circulated, and whether there are spots where the fluid is still and not being circulated, in addition to identifying any potential pitfalls or limitations for applying of the SPH method to this problem.

\section{Smoothed Particle Hydrodynamics}

In the SPH framework, the fluid is discretized into material particles that in addition to carrying the fluid properties, serve as interpolation nodes for the discretized fields. Although invented originally to simulate astrophysical problems, SPH's inherent ability to bridge the gap between continuity and fragmentation with little effort carried over well to hydrodynamical and solidmechanical applications, in addition to problems involving both, such as fluid-structure interaction, melting and solidification and industrial processing ${ }^{3,4)}$. Computationally, despite the large number of calculations 
required when compared to mesh-based methods, the high locality of SPH gives rise to ease of parallelization, which in addition to the lack of need for complicated and expensive interface-capturing techniques renders SPH attractively competitive to Eulerian CFD techniques.

For the purpose of carrying out simulations featured in this work, an in-house SPH code was written in the $\mathrm{C}++$ programing language. The open-access library Aboria was used for the general data structure and particle search algorithms ${ }^{26)}$.

\subsection{The SPH Interpolation}

A continuous field's value at a point in space can be obtained using the Dirac delta function as follows

$$
A(x)=\int A\left(x^{\prime}\right) \delta\left(x-x^{\prime}\right) d x^{\prime}
$$

Then, if the delta function is replaced by symmetric smoothing kernel, W, such that

$$
\lim _{h \rightarrow 0} W\left(\left|x-x^{\prime}\right|, h\right)=\delta\left(x-x^{\prime}\right),
$$

where $\mathrm{h}$ is the smoothing length, we end up with the SPH discretized approximation of a field value as a summation over neighboring particles

$$
\left\langle A_{a}\right\rangle=\sum_{b} \frac{m_{b}}{\rho_{b}} A_{b} W_{a b}
$$

Where $\mathrm{W}_{\mathrm{ab}}=\mathrm{W}\left(\mathrm{x}_{\mathrm{a}}-\mathrm{x}_{\mathrm{b}}, \mathrm{h}\right)$. There are several options for smoothing kernels available in the literature but in this work, the fifth order Wendland kernel is used, which is based on a family of kernels by Wendland ${ }^{4)}$. The choice of kernel is based on its low computational cost, in addition to resulting is less oscillatory and a more stable behavior with higher resolutions, when compared to other kernels. The Wendland kernel is defined as

$$
W(q)=\frac{\beta}{h^{d}}\left\{\begin{array}{cl}
(2-q)^{4}(1+2 q) & \text { for } 0 \leq q \leq 2 \\
0 & \text { for } q>2
\end{array}\right.
$$

where $q=\frac{\left\|x_{a}-x_{b}\right\|}{h}, d$ is the number of dimensions, and $\beta=7 /(64 \pi)$ in two dimensions and $21 /(64 \pi)$ in three dimensions. One of the strong suits of SPH is the ease by which the derivatives of fields can be calculated, as the derivative of the field itself is not needed, but it is calculated by means of the derivative of the smoothing kernel, which is predetermined by the choice of kernel. As such, the gradient of a scalar field can be approximated as

$$
\left\langle\nabla A_{a}\right\rangle=\frac{1}{\rho_{a}} \sum_{b} m_{b} A_{a b} \boldsymbol{\nabla} \boldsymbol{W}_{\boldsymbol{a b}}
$$

Where we adopt the following convention $\nabla W_{a b}=\frac{1}{\left\|x_{a b}\right\|} \frac{\partial W_{a b}}{\partial x_{a}}$, and $\nabla \boldsymbol{W}_{\boldsymbol{a b}}=\bar{x}_{a b} \frac{\partial W_{a b}}{\partial x_{a}}$.

The gradient can also be approximated by

$$
\left\langle\nabla A_{a}\right\rangle=\rho_{a} \sum_{b} m_{b}\left(\frac{A_{a}}{\rho_{a}^{2}}+\frac{A_{b}}{\rho_{b}^{2}}\right) \nabla \boldsymbol{W}_{\boldsymbol{a} \boldsymbol{b}}
$$

As Monaghan pointed out, the gradient approximation in Eq. 6 has the advantage of conserving linear and angular momentum, and is therefore the form of choice in the

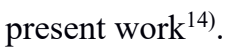

\subsection{SPH Discretization of the Navier-Stokes Equation}

Using the SPH interpolation to discretize the Lagrangian form of the momentum equation we have

$$
\left\langle\frac{d u_{a}}{d t}\right\rangle=-\sum_{b} m_{b}\left(\frac{p_{a}}{\rho_{a}^{2}}+\frac{p_{b}}{\rho_{b}^{2}}\right) \nabla \boldsymbol{W}_{\boldsymbol{a} \boldsymbol{b}}+\left\langle(\nabla \cdot v \nabla) u_{a}\right\rangle
$$

where the second term on the right of Eq. (7) is the SPH approximation of viscosity for which there are several options in the literature. In this work we use the SPH viscosity formulation by Morris ${ }^{5)}$.

$$
\left\langle(\nabla \cdot v \nabla) u_{a}\right\rangle=\sum_{b} m_{b} \frac{\left(\mu_{a}+\mu_{b}\right) u_{a b}}{\rho_{a} \rho_{a}} \nabla W_{a b}
$$

The density is calculated by means of solving the conservation of mass, or continuity equation, discretized in SPH as

$$
\left\langle\frac{d \rho_{a}}{d t}\right\rangle=\sum_{b} m_{b} u_{a b} \cdot \nabla W_{a b}
$$

In this work, the weakly compressible formulation of SPH is adopted, where pressure is computed by means of an equation of state

$$
p_{a}=\rho_{0}\left[\left(\frac{\rho}{\rho_{0}}\right)^{\gamma}-1\right]
$$

Where $\gamma=7$, and the zero subscript denotes reference initial values.

To reduce the order of density fluctuations, the density field is reset every 40 iterations using the so-called Shepard filter as follows ${ }^{6}$.

$$
\rho_{a}=\frac{\sum_{b} m_{b} W_{a b}}{\sum_{b} \frac{m_{b}}{\rho_{a}} W_{a b}}
$$

The XSPH correction is used to reduce the divergence of a particle's velocity from the average velocity in its vicinity, where the XSPH term is added to the velocity of each particle

$$
X S P H=\varepsilon \sum_{b} \frac{m_{b}}{\bar{\rho}_{a b}} u_{a b} W_{a b}
$$

$\varepsilon$ being a tunable parameter between 0 and 1 .

\subsection{Solid Boundary Treatment}

Boundary treatment is known to be one of the most challenging aspects of the SPH method, since they do not arise naturally from the formal SPH discretization ${ }^{9}$, making boundary treatment a major area of research in the SPH community in the past three decades.

One of the earliest and most widely solid boundary implementations in SPH is the method of virtual or ghost particles, where fictitious particles are placed outside of 
the solid boundary that mirror the velocity of any fluid particles within the smoothing length of the boundary particles, but possess the same density and pressure ${ }^{7)}$. This method helps mitigate the issue of "kernel truncation" that reduces the accuracy of the SPH approximation near boundaries of the fluid when compared to its interior, due to the scarcity of particles within the support domain of the fluid particle in question. However, the constantly changing number of virtual boundary particles increases implementation complexity and computational cost especially for complicated geometries and high resolutions.

The particle nature of SPH has also inspired the adoption of inter-particle repulsive forces, analogous to the repulsive forces that arise when neutral atoms or molecules interact, to enforce solid wall boundary conditions.

The most popular way of enforcing solid boundaries by means of such inter-particle repulsive forces is the Lennard-Jones potential, where a fluid particle experiences a radially directed repulsive force inversely proportional to its distance from a boundary particle. Repulsive force boundary formulations, although relatively easy to implement, may lead to spurious particle motion near the boundary as it fails to compensate for kernel truncation ${ }^{8)}$.

In this work, the so-called "dynamic boundary condition" is used to model solid boundaries ${ }^{10)}$.

In the dynamic boundary treatment, particles are placed at the boundary and are subjected to the same treatment as fluid particles, with the exception of having a predetermined positions and velocities. Since boundary particles are governed by the same momentum and continuity equations as the fluid, when fluid particles approach the boundary, the pressure and density increases, consequently repelling fluid particles and preventing fluid-boundary penetration. Also, the viscous term in the momentum equation enforces the no-slip condition at the boundary ${ }^{10)}$.

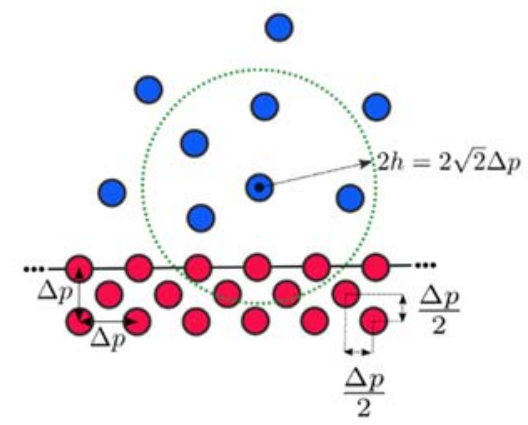

Fig. 1: Staggered configuration of dynamic boundary particles (red) and fluid particles' (blue) support domain (green dashed circle)

In addition to being convenient, the ability to perform inter-particle interaction computations of the boundary particles in the same loop as the fluid particles has the advantage of improving computational efficiency and parallelization. This, however, allows pressure waves to travel across the boundary, inducing spurious and undesirable fluctuations in pressure and free-surface profile near the boundary; it was therefore found best to prohibit inter-boundary-particle interactions in the simulation, and alleviate the resulting discontinuity of pressure values by filtering their density in the same manner as fluid particles. In order to minimize kernel truncation error near the boundary and prevent particle penetration at high fluid-solid wall impact velocities, 3 rows of dynamic particles are placed at the boundary in a staggered manner.

Fig. 1 illustrates the manner in which boundary particles are arranged, where $\Delta \mathrm{p}$ is the initial particle spacing. The radius of the circle or sphere on which the SPH interpolation is carried out, is $2 \mathrm{~h}$, where

$$
h=\sqrt{2} \Delta p
$$

\subsection{Open Boundary Treatment}

Fig. 2 below shows an illustration of the implementation of the inlet boundary treatment used in this work.

The structure of buffer zones adopted by Tafuni et. al is used to prevent kernel truncation for fluid particles near the boundary ${ }^{11)}$.

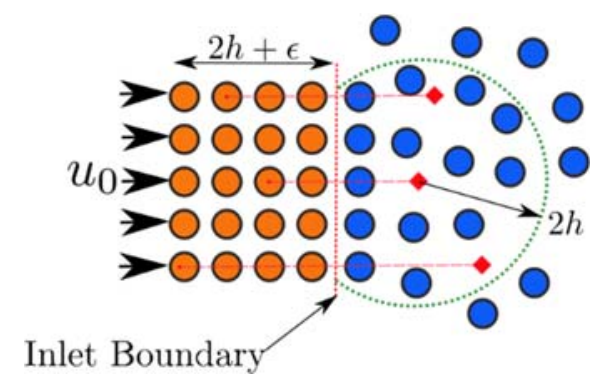

Fig. 2: Inlet buffer particles (orange), whose pressure is interpolated from ghost nodes (red diamonds) before crossing the inlet boundary and becoming regular fluid particles (blue).

A buffer layer of particles is created with a thickness of $2 h+\epsilon$, where $\epsilon$ is a small number to ensure the inclusion of all buffer particles in the support domain of particles near the boundary.

Inlet buffer particles are created in a regular lattice and assigned with the inlet velocity, $\mathrm{u}_{0}$, in the direction normal to the inlet boundary. The position of the buffer region particles is simply marched in time towards the inlet boundary, and when an inlet buffer particle crosses the boundary, it is converted to a fluid particle and another buffer particle is created in its place at the beginning of the buffer.

Simply assigning a fixed pressure to the buffer particles was found to create unphysical fluctuations in velocity and pressure near the boundary and eventually lead to a crash in the simulation. In order to mitigate such spurious behavior and better enforce the natural, or zero gradient, pressure boundary condition at the inlet boundary, the density, and therefore pressure, of inlet buffer particles is interpolated from within the fluid domain by means of 
interpolation ghost nodes as shown in Fig. 2. The position of the ghost nodes is found by mirroring the position of the buffer particles about the inlet boundary, and the density at the node is found by using the Shepard filter given by Eq. 7, but without including other buffer particles in the interpolation to minimize dissipation. The pressure is then calculated by means of Eq. 6 .

As regards the outlet boundary treatment, simply deleting particles that cross the outlet results in pressure and velocity fluctuations and pressure waves reflecting off the boundary, resulting in unphysical behavior and artifacts in the solution. To avoid such issues, the buffer zone approach is also used to model the outlet boundary condition as shown in Fig. 3. Once the fluid particles cross the outlet boundary, they are converted into outlet buffer zone particles, and their pressure is set to the prescribed outlet pressure, while their density is frozen. The particles' velocity component that is orthogonal to the boundary normal is zeroed once particles cross the boundary. The position of the outlet buffer zone particles are marched with time until they cross the end of the buffer zone, where they are marked for deletion at the next time step.

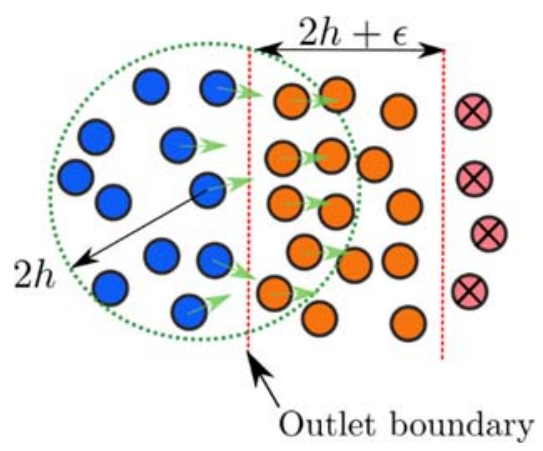

Fig. 3: Fluid particles (blue) are converted to outlet buffer particles (orange) when they cross the outlet boundary, then they are marked for deletion (red) once they leave the buffer zone

\subsection{Time Integration}

Geometric, or symplectic time integrators are often used in particle-based physics simulations due to their ability to exactly preserve the Lagrangian, and therefore the total energy of the system ${ }^{14}$. Although higher order time integrators, such as the Runge-Kutta method, are superior in terms of absolute accuracy, geometric integrators' ability to preserve angular momentum yield time-reversible and more stable results ${ }^{14}$.

In this work, the second-order symplectic time-stepping scheme is used.

First, the position, velocity and density are predicted by advancing half a time-step as follows

$$
\begin{gathered}
x^{1 / 2}=r^{0}+\frac{\Delta t}{2} u^{0} \\
u^{1 / 2}=u^{0}+\frac{\Delta t}{2} F\left(x^{-\frac{1}{2}}, u^{-\frac{1}{2}}, \rho^{-1 / 2}\right)
\end{gathered}
$$

$$
\rho^{1 / 2}=\rho^{0}+\frac{\Delta t}{2} D\left(x^{-1 / 2}, u^{-1 / 2}\right)
$$

where $\mathrm{F}$ is the momentum equation and $\mathrm{D}$ is the continuity equation. Finally, the variables are corrected by advancing one more half a time-step, using the continuity and momentum equations evaluated with the predicted values in the previous stage of the integration scheme

$$
\begin{gathered}
u^{1}=u^{0}+\Delta t F\left(x^{1 / 2}, u^{1 / 2}, \rho^{1 / 2}\right) \\
x^{1}=r^{1 / 2}+\frac{\Delta t}{2} u^{1} \\
\rho^{1}=\rho^{1 / 2}+\frac{\Delta t}{2} D\left(x^{1}, u^{1}\right)
\end{gathered}
$$

then the pressure's equation of state is evaluated using the corrected density at the end of the time step.

The size of the time-step used in the integration scheme is updated every time-step to ensure numerical stability according to the Courant-Friedrichs-Lewy (CFL) condition and criteria depending on the force per unit mass of fluid the and viscous diffusion term, as per the work done by Monaghan and $\operatorname{Kos}^{15}$.

The minimum time step due to the force is computed as follows

$$
\Delta t_{F}=\min \left(\sqrt{\frac{h}{\left\|F_{a}\right\|}}\right)
$$

where $F_{a}$ is the force per unit mass evaluated for all particles. The time-step according to the CFL criteria and viscous diffusion is

$$
\Delta t_{C F L, v}=\min \left(\frac{h}{c_{s}+\max _{b}\left|\frac{h u_{a b} \cdot x_{a b}}{x_{a b}^{2}+\epsilon}\right|}\right)
$$

where $c_{s}$ is the speed of sound, set to be 10 times the maximum velocity anticipated in the simulation, and $\varepsilon=$ $0.01 \mathrm{~h}^{2}$. The time-step is then found as follows

$$
\Delta t=\alpha \min \left(\Delta t_{F}, \Delta t_{C F L, v}\right)
$$

$\alpha$ being a factor of safety set to 0.3 in this work.

\section{Dam-Break Validation}

Dam-break, or the collapse of a water column, is one of the most widely studied phenomena in computational fluid dynamics due to the presence of highly non-linear phenomena and violent fluid-boundary interactions, and its similarity to flow regimes that occur in naval and coastal engineering. The numerical simulation of dambreak problems have been extensively studied using both Lagrangian and Eulerian methods, and even statistical mechanical methods such as the Lattice-Boltzmann method. Example of such works are the Finite-Volume simulations by Kamra et. al and the Lattice-Boltzmann simulations by Nik et. $\mathrm{al}^{12,19)}$. In order to validate the SPH 
implementation in this work, the dam break flow over a dry bed experiment by Lebovsky et. al ${ }^{16)}$ is simulated in two dimensions, and the water level, propagation of the wave front, pressure and free surface profile are compared with the experimental measurements. Fig. 4 below shows a sketch of the initial configuration and measurement locations.

Fig. 5 shows satisfactory agreement between the pressure measurement on the left wall from experiment and the SPH simulation. In the SPH simulation, the effect of the gate motion, which is used to release the fluid and initiate the flow, has not been accounted for, resulting in an early time of impact in the simulation when compared to the experiment as has been noted by Kamra ${ }^{17}$.

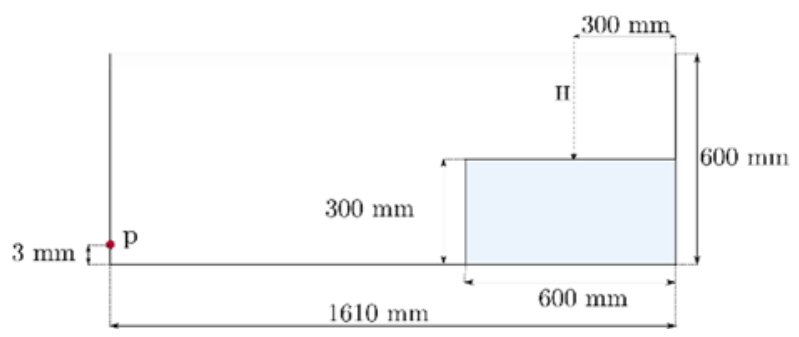

Fig. 4: Sketch of the initial configuration of the 2dimensional dam break simulation showing the pressure measurement point (red dot) and the water elevation $(\mathrm{H})$ measurement location
In Fig. 6, adequate agreement is found between the water elevation obtained from the simulation and the experimental measurements by Lobovsky et. al.

Fig. 7 compares the evolution of the wave front's nondimensional location vs. time obtained from SPH simulation and the experiments by Lobovsky et. al and $\mathrm{Hu}$ and Sueyoshi ${ }^{18)}$. The deviation between numerical and experimental results is consistent with what is expected when the gate effect is not accounted for, as found by Suyoshi and $\mathrm{Hu}^{19)}$, and is comparable to Finite Volume simulations done by Kamra attempting to quantify extent to which the gate effect can alter the flow ${ }^{17}$.

A comparison between the evolution of the free surface in the experiment and simulation is shown in Fig. 8. The pictures of the free surface from the Lobovsky experiment are snapshots from a video.

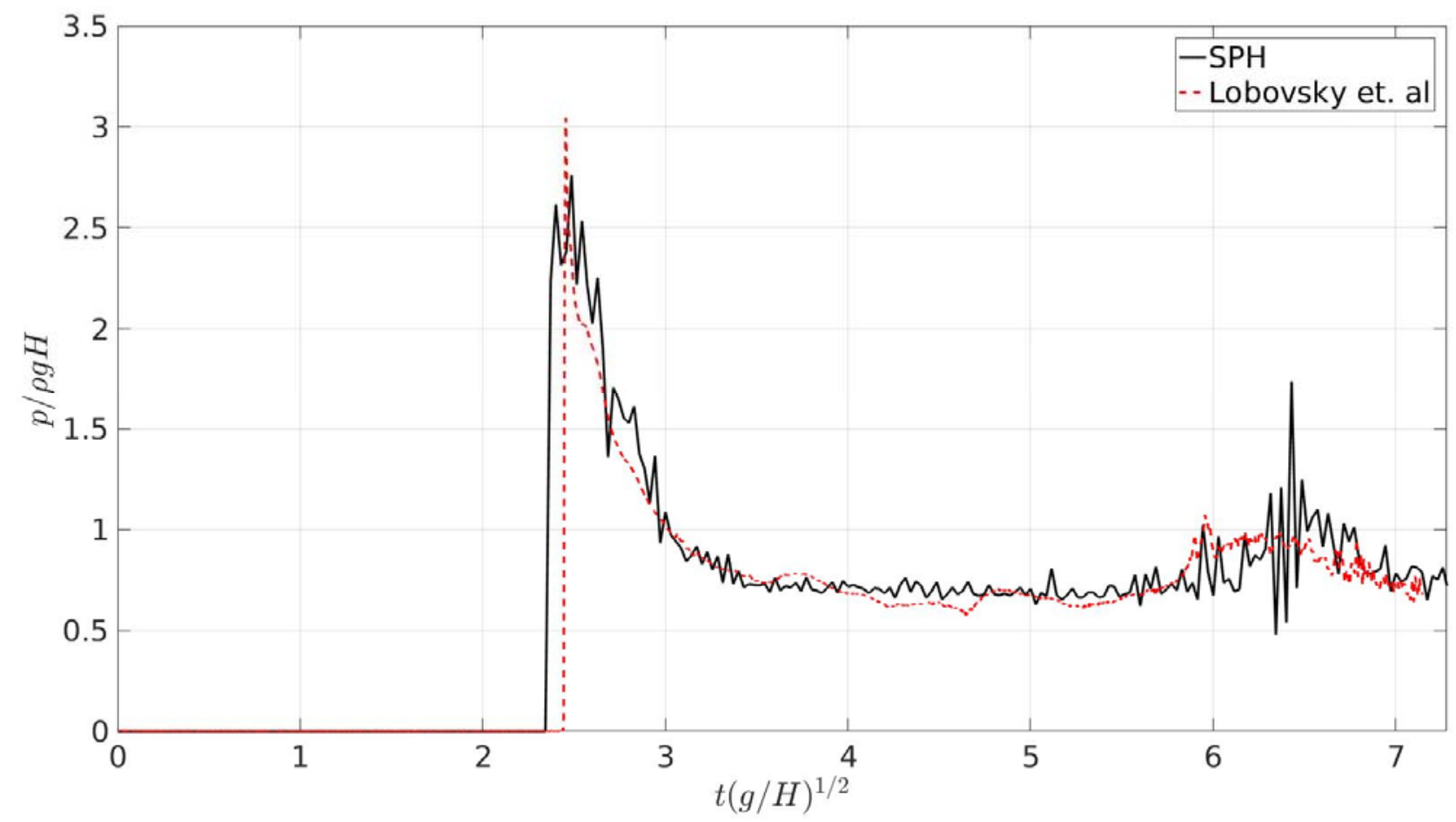

Fig. 5: Pressure from SPH simulation vs. pressure measurement by Lobovsky et. al. 


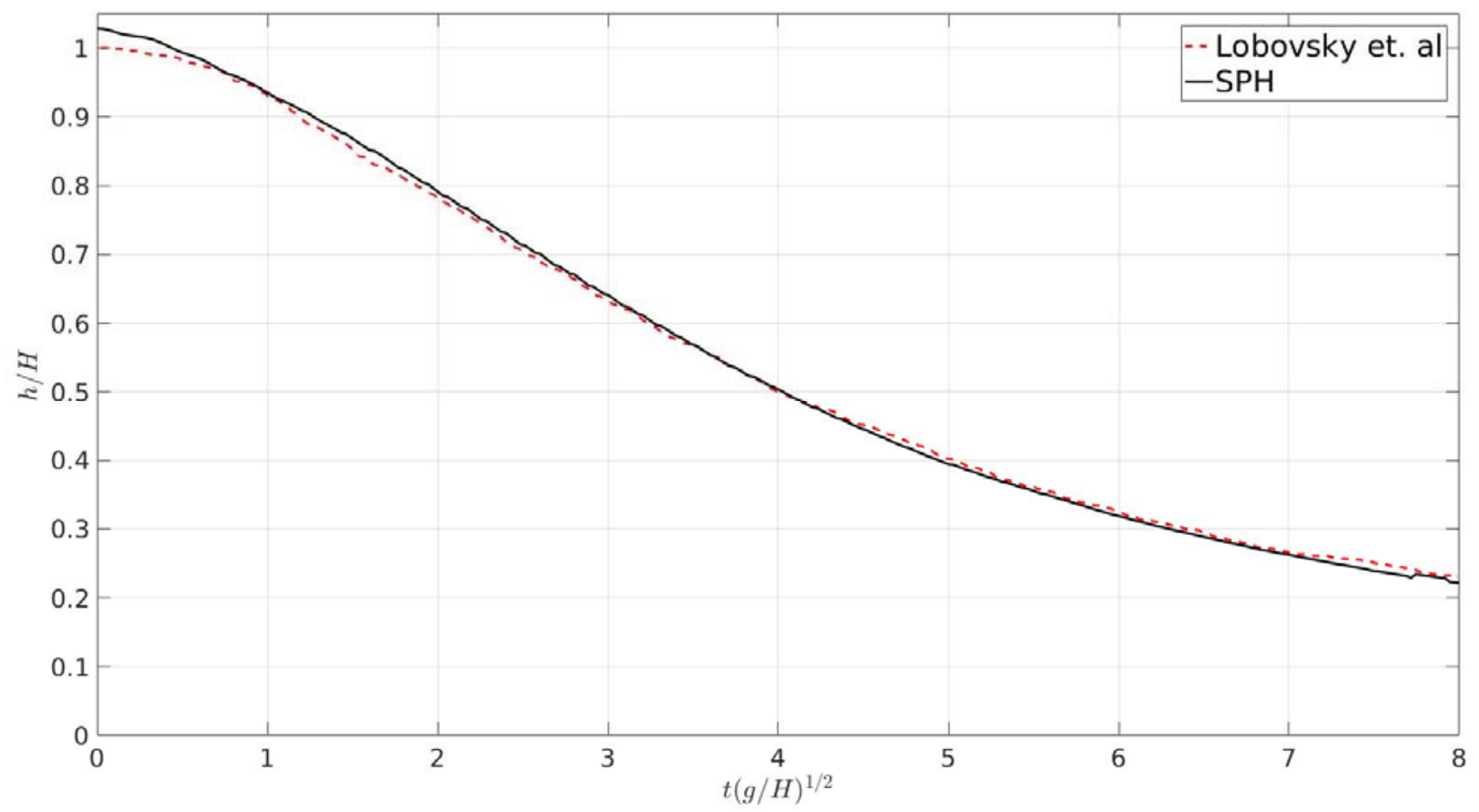

Fig. 6: Water level from SPH vs. experiment by Lobovsky et. al

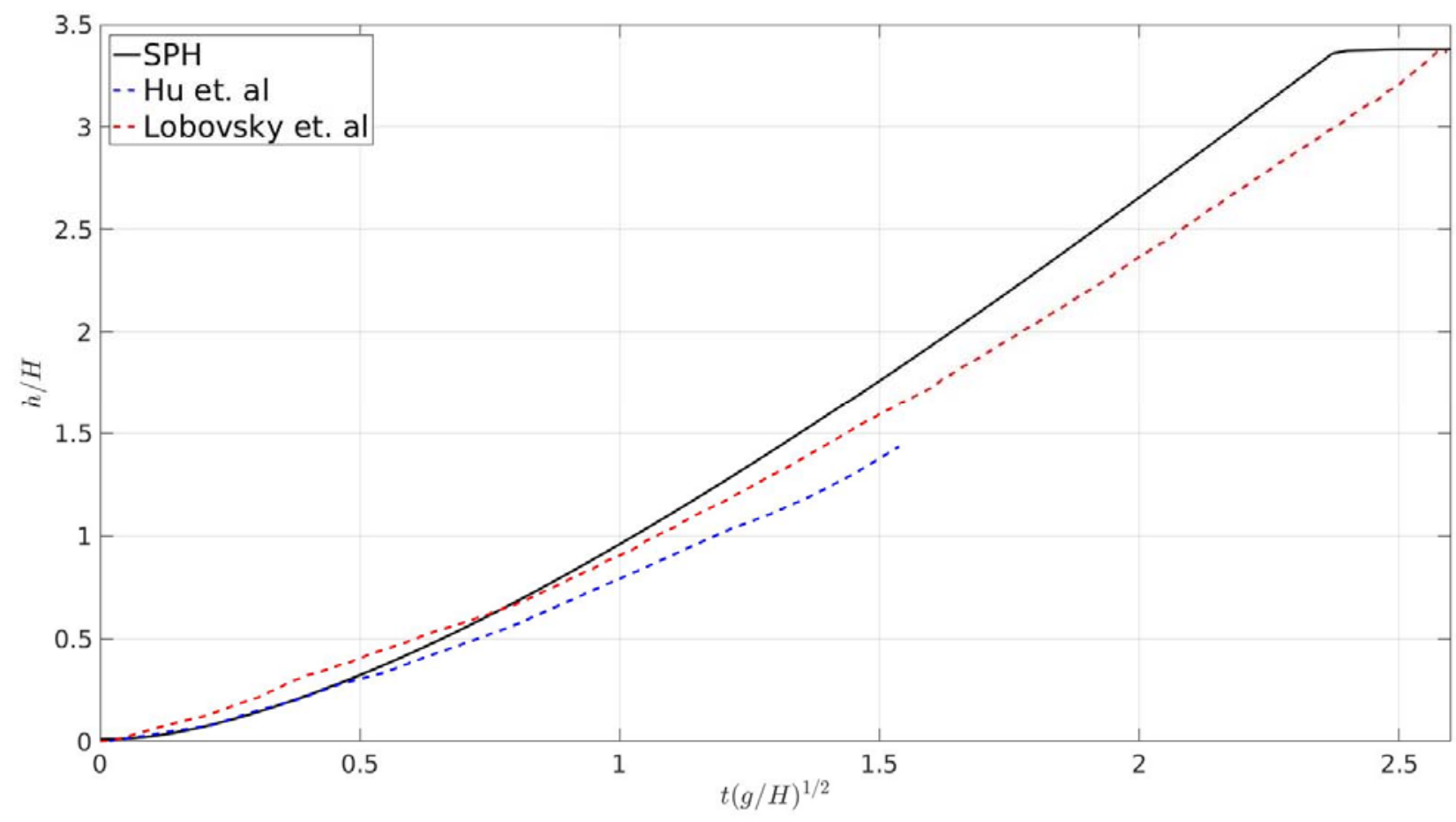

Fig. 7: Wave front propagation vs. dimensionless time from SPH compared to measurements from experiments by $\mathrm{Hu}$ et. al and Lobovsky et. al 


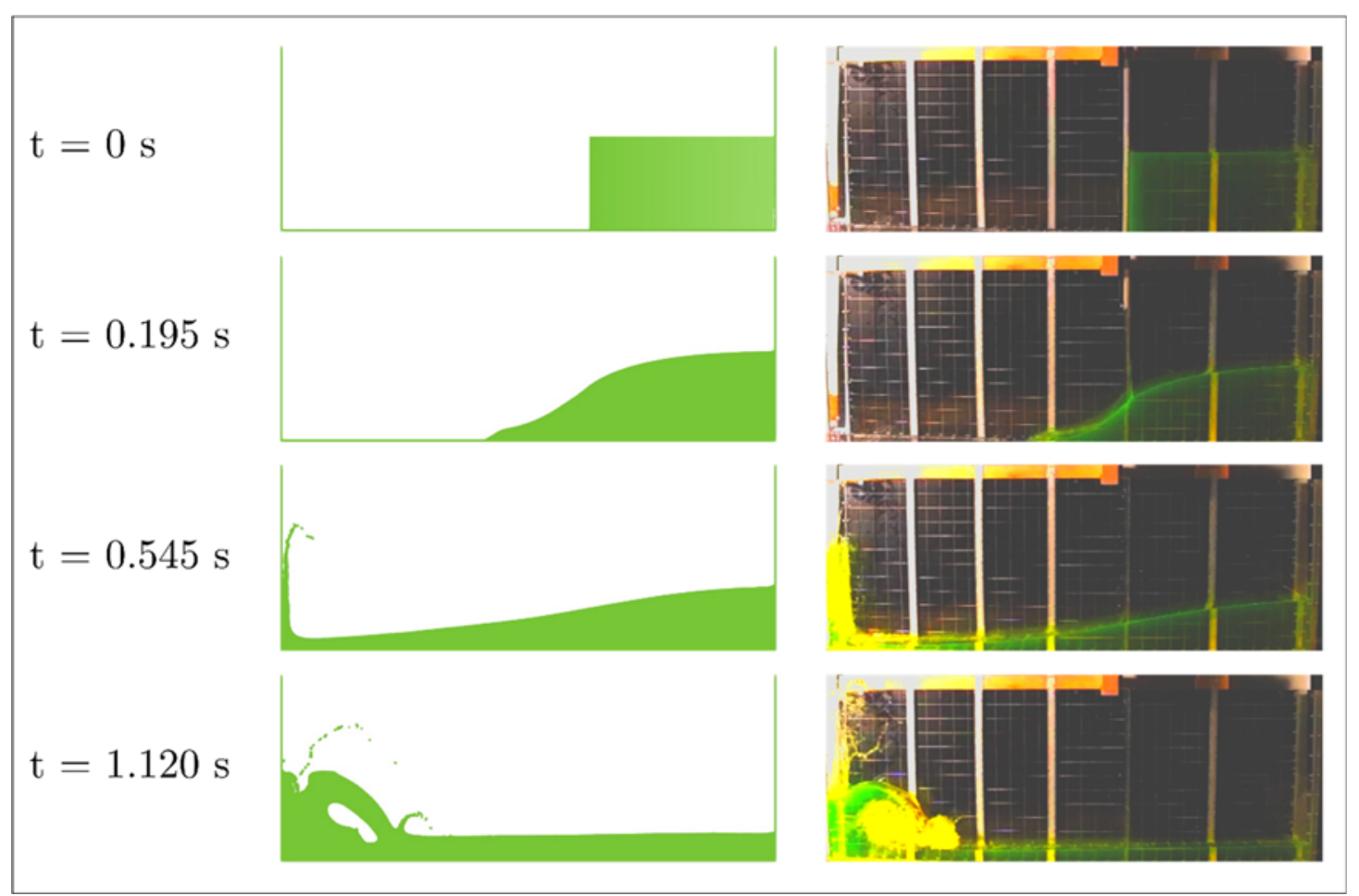

Fig. 8: Free surface snapshots of the dam break SPH simulation (left) vs. experiment by Lobovsky et. al (right)

\section{Liquid Metal Divertor Simulation}

Fig. 9 below shows a sketch of the front view of a tank initially partially filled with liquid metal. The physical parameters used for the liquid metal in the simulation are those of GaInSn, a candidate for use as liquid metal divertor material. Table 1 shows some of the physical parameters that were used in the simulation ${ }^{16)}$.

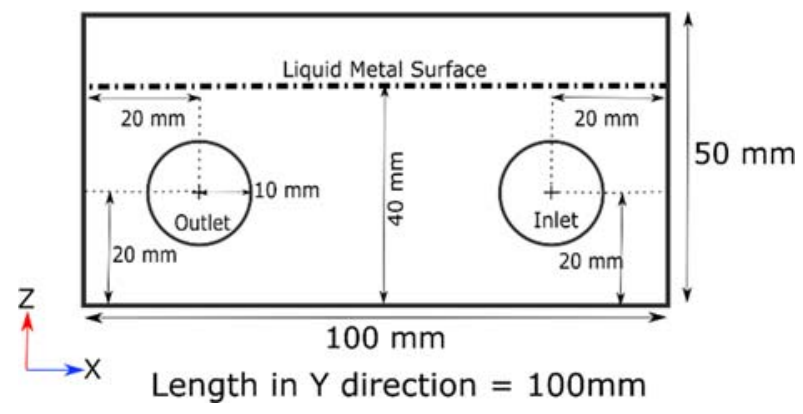

Fig. 9: Sketch of the numerical liquid metal divertor tank
Table 1: Some physical properties of GaInSn

\begin{tabular}{|l|l|}
\hline Melting point $\left({ }^{\circ} \mathrm{C}\right)$ & 10.5 \\
\hline Ga $(\% \mathrm{vol})$ & 67 \\
\hline In $(\% \mathrm{vol})$ & 20.5 \\
\hline Sn $(\% \mathrm{vol})$ & 12.5 \\
\hline Density $\left(\mathrm{Kg} / \mathrm{m}^{3}\right)$ & 6360 \\
\hline Viscosity $\left(\mathrm{m}^{2} / \mathrm{s}\right)$ & $2.98 \times 10^{-7}$ \\
\hline
\end{tabular}

In the simulation of the liquid metal divertor, the tank was initially partially filled with fluid, so that the free surface was $40 \mathrm{~mm}$ high. The velocity of the liquid metal entering the tank through the inlet was set to $0.25 \mathrm{~m} / \mathrm{s}$, or $0.0785 \mathrm{l} / \mathrm{s}$ and the pressure at the inlet was interpolated from inside the domain, as per section 2.3 in this paper. The velocity at the inlet was chosen to avoid completely draining the tank or overtopping and the pressure at the outlet was set to zero. Fig.10 and Fig.11 show the free surface evolution, distribution of the new fluid particles introduced to the domain through the inlet and the velocity vector field on a plane parallel to the $\mathrm{x}-\mathrm{y}$ plane, at a height of $20 \mathrm{~mm}$. The free surface shown in the figures below was captured by means of the SPH first moment, since it is approximately equal to 1 in the interior of the domain and its value drops near the free surface, as follows:

$$
\left[\sum_{b} \frac{m_{b}}{\rho_{a}} W_{a b}\right]_{\text {free surface }} \leq 0.80
$$




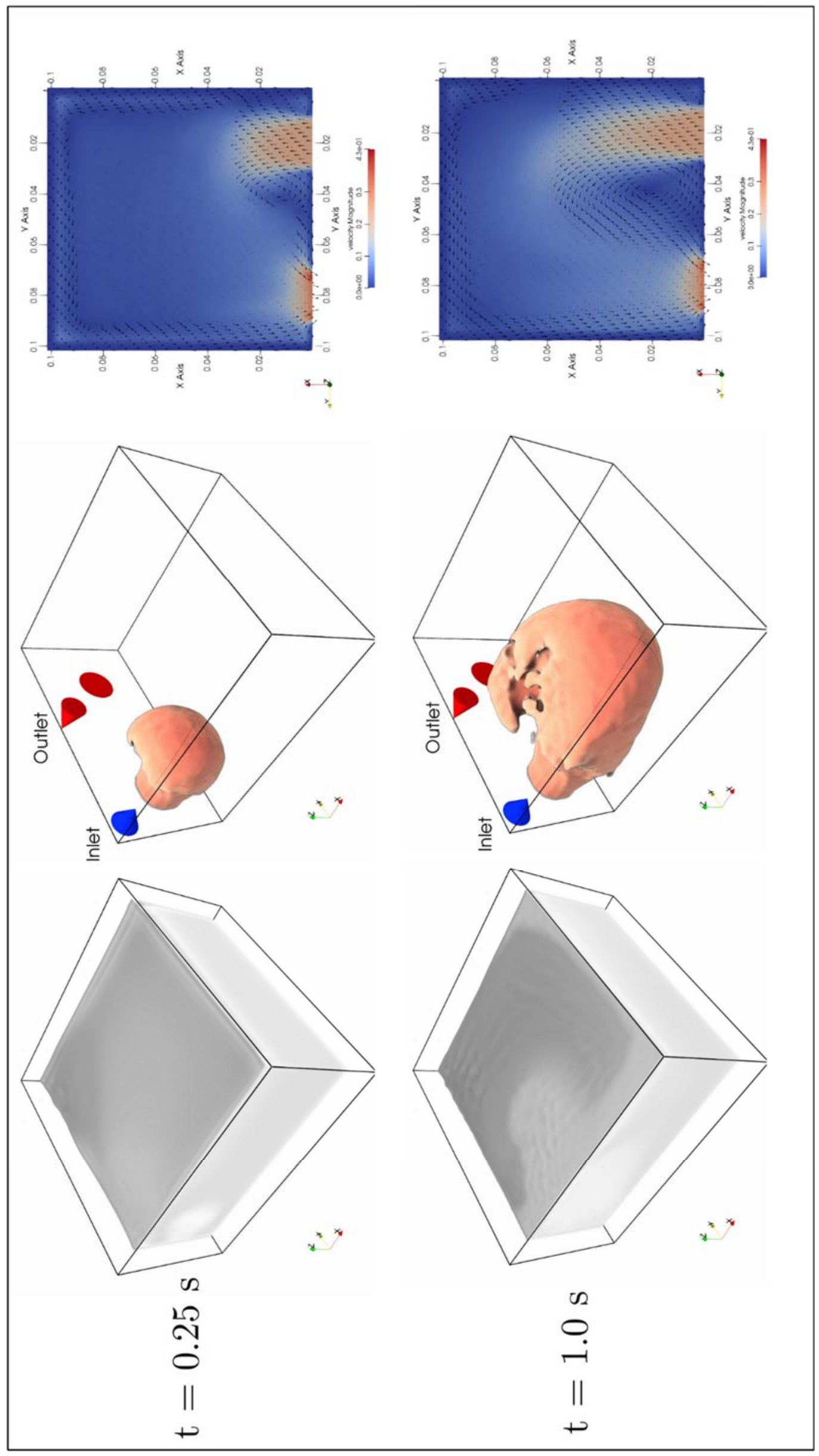

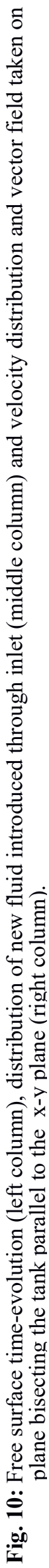




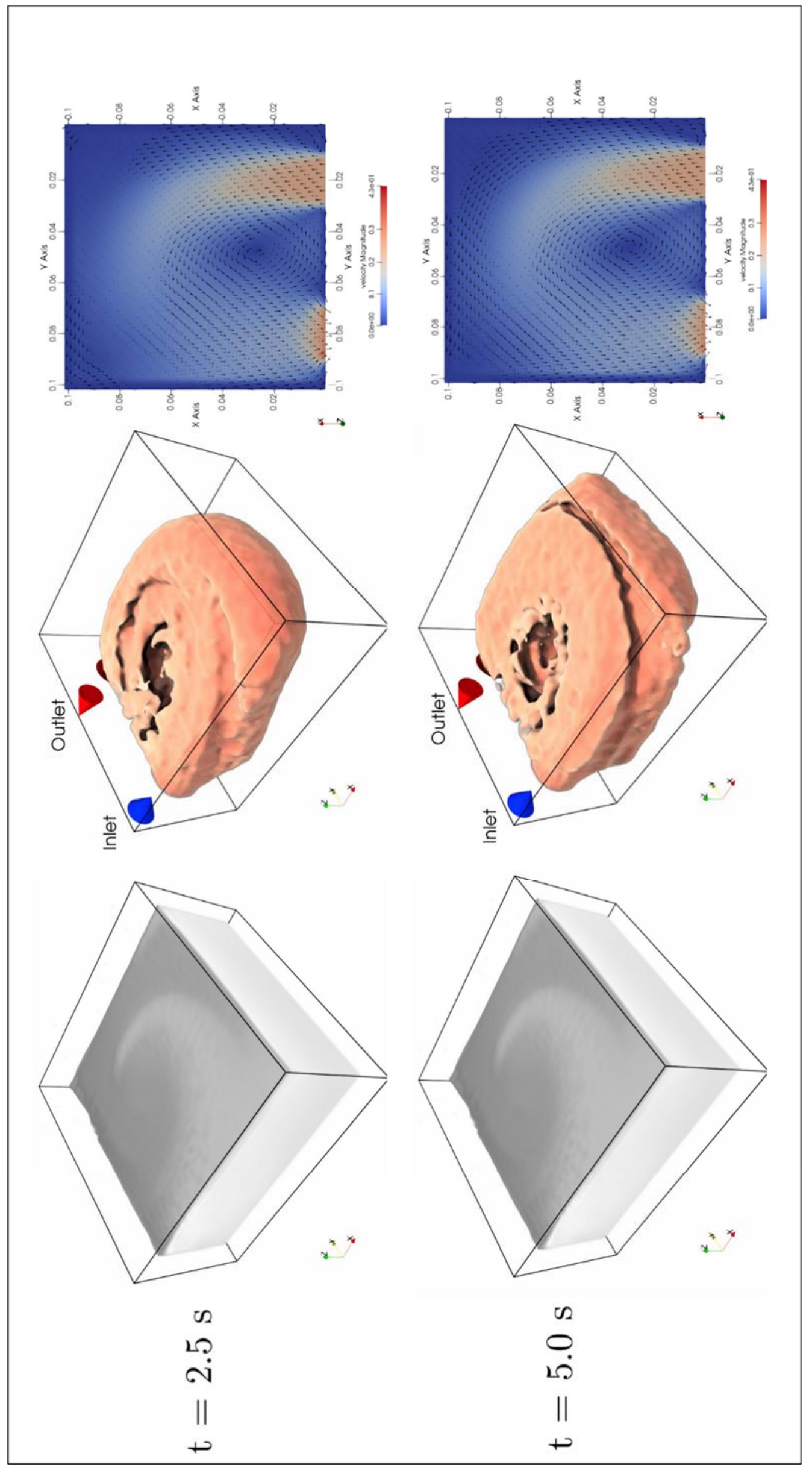

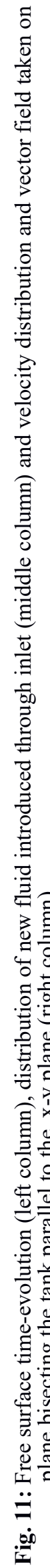


The simulation shows that in areas around the corners of the tank opposite to the inlet and outlet in addition to the area in the center of the tank fluid is not circulated sufficiently which may result in reduced heat removal efficiency. Simply increasing the velocity at the inlet to attempt to induce more highly turbulent flow patterns in the tank may result in overtopping, therefore additional solid structures inside the tank that aim to enhance mixing and circulation may be needed. The flow pattern may be significantly altered by the presence of a magnetic field, which was not accounted for in this work. An accurate and comprehensive simulation of all physical aspects relevant to the operation of the liquid metal divertor necessitates the inclusion of magneto-hydrodynamic effects, which is part of planned future work.

\section{Conclusion and Future Work}

This paper aimed to lay the groundwork for running multi-physics simulations of liquid metal divertors using Smoothed Particle Hydrodynamics by means of first instigating a hydrodynamically accurate implementation of SPH that can then be used to simulate free-surface problems. In this paper, Smoothed Particle Hydrodynamics formalism was introduced and then it was shown how it could be used to discretize the NavierStokes equations to simulate hydrodynamical problems. The details of the SPH implementation used in simulations shown in this work were detailed and then validated by simulating a dam break problem and comparing the results with experimental measurements from the literature. The validation case yielded satisfactory matching with the experimental results. A simplified and scaled model of the liquid metal divertor tank was then simulated and some potential pitfalls of the suggested design were identified.

In order to capture the full dynamics of the divertor in a fully realistic and accurate manner, the addition of magneto-hydrodynamics, surface tension and turbulence modes is necessary and planned as part of future work. Furthermore, to be able to qualify and evaluate the practicality and effectiveness of different liquid metal divertor designs for use in nuclear fusion reactors, simulating the magnetohydrodynmic and heat transfer physics of the problem is essential, as such effects may significantly alter the flow pattern.

\section{References}

1) S. V. Mirnov, V. N. Dem'yanenko, E.V. Murav'ev, J. of Nuclear Material, 196-198, 105 (1992).

2) T. W. Morgan, P. Rindt, G. G. van Eden, V. Kvon, M. A. Jaworksi, N J. Lopes Cardozo, Plasma Phys. and Controlled Fusion, 60, (2017).

3) C. Alvarado-Rodriguez, J. Klapp and L. Sigalotti, Computers and Fluids, 159, p. 177 (2017).

4) H. Wendland, Adv. in Comp. Math., 4, p. 389 (1995)
5) J. Morris, P. Fox, Y. Zhu, J. of Comp. Phys., 136, p. 21 (1997).

6) D. Shepard, ACM Nat. Conference, 23, p. 517, New York (1968).

7) J. C. Crespo, M. Gomez-Gesteira, R. A. Dalrymple, Comput. Mater. Contin., 5, p. 173 (2007).

8) P. W. Randles, L. D. Libersky, Comput. Meth-ods Appl. Mech. Eng., 138, 375 (1996).

9) M. Ferrand, D. R. Laurence, B. D. Rogers, D. Violeau, and C. Kassiotis, Int. J. Numer. Methods Fluids, 71, p. 446 (2013).

10) J. C. Crespo, M. Gomez-Gesteira, R. A. Dalrymple, Comput. Mater. Contin., 5, p. 173 (2007).

11) Tafuni, I. Sahin, and R. Vacondio, Spheric, Munich (2016).

12) M. Kamra, C. Hu, Evergreen, 4, p.25, (2017).

13) J. Monaghan, Reports Prog. Phys., 68, p. 1703 (2005).

14) J. Monaghan, A Kos, Coastal and Ocean Engineering, 125, p. 145 (1999)

15) N. Morely, J. Burris, L. Cadwallader, M. Norenberg, Rev. of Sci. Instruments, 79, (2008).

16) L. Lobovský, E. Botia-Vera, F. Castellana, J. MasSoler, and A. Souto-Iglesias, J. Fluids Struct., 48, p 407 ( 2014).

17) M. Kamra, Kyushu University, PhD thesis (2018).

18) $\mathrm{Hu}$ and M. Sueyoshi, Jour. of Mar. Sci. and App. 9, p. 109 (2010).

19) N. Mohd, M. Kamra, M. Sueyoshi, C. Hu, Evergreen, 4, (2017).

20) A. Y. Ying, M. A. Abdou, N. Morley, T. Sketchley, R. Woolley, J. Burris, and M. Narula. Fusion Eng. and Design, 72, p. 35-62, (2004).

21) M. Shimada and Y. Hirooka. Nuclear Fusion, 54, p.122002 (2014).

22) C. Liao and M. S. Kazimi. Fusion Technology, 21, p. 1845-1851 (1992).

23) M. S. Shadloo, G. Oger, and David Le Touzé. Computers \& Fluids, 136, p. 11-34 (2016).

24) C. Ulrich, M. Leonardi and T. Rung. Ocean Engineering, 64, 109-121 (2013).

25) J. Hall, T. C. S. Rendall, C. B. Allen, and H. Peel. Journal of Fluids and Structures, 56, p. 11-32 (2015).

26) M. Robinson and M. Bruna. SoftwareX, 6, p. 172-178 (2017). 